\title{
A2CDC: Area Coverage, Connectivity and Data Collection in wireless sensor networks
}

\author{
Garrik Brel Jagho Mdemaya $^{1}$ and Alain Bertrand Bomgni ${ }^{2}$ \\ Dept. of Mathematics and computer science, University of Dschang \\ PO Box 67, Dschang, Cameroon \\ 1jaghobrel@gmail.com, 2alain.bomgni@gmail.com
}

Received: Sep. 29, 2018 Accepted: December 26, 2018 Published: December 31, 2018

DOI: 10.5296/npa.v10i4.13977

URL: https://doi.org/10.5296/npa.v10i4.13977

\begin{abstract}
Wireless sensor networks are increasingly being deployed in areas where several types of information need to be harvested. Monitoring a given area is one of the main goals of this technology. This consists in deploying sensor nodes in the Area of Interest (AoI) in order to detect any event occurring in this area, collect information and send them to the base station. However, in this type of configuration, the quantity and the quality of data collected are important factors in making better decisions by the end user. It therefore becomes crucial to deploy sensors in the AoI so that the latters can cover as much as possible the AoI, and propose mechanism to collect and send data to the base station while minimizing the energy consumption of the sensors. In this paper, we bring into focus a solution "Area Coverage, Connectivity and Data collection in wireless sensor networks (A2CDC)" to resolve this problem which performs in two main stages: in the first stage, we propose an algorithm that guarantees a maximal coverage of the AoI after a random deployment of static sensors and mobile sensors; and in the second stage, we propose a node activity scheduling that minimizes energy consumption of both static and mobile nodes while sending collected data to the base station. Compared to many other algorithms in the literature, our solution is better in term of coverage percentage of the AoI, data received by the base station and in term of energy minimization.
\end{abstract}

Keywords: Area coverage, Wireless sensor network, Sensors deployment problem, energy consumption 


\section{Introduction}

Since their creation, wireless sensor networks have continued to grow in popularity in the scientific and industrial communities [1]. With their various advantages, this technology has established itself as a key player in today's communication network architectures [1]. A wireless Sensor Network (WSN), which is a targeted wireless network, consists of a significant number of miniaturized electronic devices, called sensors, distributed over a specified area in order to sense the environment and communicate the accumulated information from the monitored field to other networks (e.g., the internet) [2]. These networks have been extensively used for monitoring of various physical or environmental conditions [3]. A network of sensors suffers from several technical weak points such as communication range, monitoring range, low battery, and network deployment circumstance problems such as the difficulty of building a sensor network in volcanoes, mountains, or in the oceans [4]. Sensor deployment can either be deterministic or random. In deterministic deployment, coverage can be maximized as a result of optimal placement of sensor nodes. Random deployments are preferred when the region information is not known apriori [5].

In such systems, maximum coverage of the AoI and full connectivity between the deployed nodes are two important factors in sending as much good quality information as possible to the end user through the base station for better decision-making. This can be illustrated when resolving problems like detecting and tracking of intruders in restricted areas. Such applications require full area coverage; furthermore, the most critical zones should be covered by more than one sensor node. Permanent connectivity is also required [6]. Various works have been done in the literature in order to solve this type of problems after deployment of sensor nodes. Some of them consider random deployment; others consider a deterministic deployment while others consider both. In this paper, we propose a method to cover as much as possible the AoI using both static and mobile sensors while ensuring full connectivity between the deployed nodes. After random deployment of static nodes, we perform an algorithm which helps us to find empty clusters (holes) in the network. We therefore use mobile nodes in order to cover these holes. Mobile nodes are also randomly deployed, but we propose an algorithm to move them in order to feed empty clusters. This approach is followed by an algorithm of scheduling node activity that minimizes the energy consumption of the nodes while collecting and sending data to the base station. In fact, our solution performs one algorithm for normal nodes which send data to their cluster-heads $(\mathrm{CH})$ and another algorithm for $\mathrm{CH}$ which send data to the base station.

The remainder of this paper is organized as follow: in section 2 we present the various works dealing with the deployment, coverage and connectivity problems; in section 3, we describe our contribution, then in section 4 we present differences between our protocol and some others protocols existing in literature, section 5 deals with some experimental results. A conclusion with open problems ends the paper.

\section{Related works}

Several works have been done in order to introduce basic concepts related to coverage and connectivity ([6], [7], [8], [9], [10] and [11]). Each of them addresses coverage and 
connectivity problem differently; but in [6], a better description of this problem is done and authors separate the coverage problem in three types of coverage: Area coverage, barrier coverage and point coverage.

\subsection{Area coverage and connectivity}

The goal in the area coverage problem is to cover the whole area. Therefore, in some cases, the number of sensors is not sufficient; the goal of area coverage becomes maximizing the coverage rate. Works intended to resolve area coverage and connectivity problem are massively done. In [4] authors achieve both random and deterministic deployment in order to cover as much as possible the area of interest. After deployment, they propose a random node activity scheduling which relies on a random number $P_{i}$ that helps to determine the next node to be activated to monitor information in a cluster. Thus a node whose residual energy is finished can be chosen to be activated and, since this node is the one that has to select the next node to be activated in a cluster using $\mathrm{P}_{\mathrm{i}}$, this cluster can be paralyzed and sensors in this cluster won't be able to collect information anymore. Connectivity between sensors of this cluster and sensors of the others clusters is therefore impossible. [12] proceeds to a random deployment of static nodes and thereafter, proceeds to deployment of some mobile nodes that are used to repair the coverage holes after initial deployment of the static nodes. This solution ensures a good coverage ratio but not connectivity between sensors. [13] and [14] proposed algorithms that guarantees full coverage and multiple connectivity [6] after regular sensors deployment. But these solutions assume that the AoI is regular. In [2], a deployment approach based on flower pollination algorithm (FPA) was proposed. This approach can find the optimal placement topology in terms one QoS metric and ensures simple connectivity between sensors but it did not incorporate other QoS metrics like energy consumption.

\subsection{Barrier coverage and connectivity}

Wireless sensors networks are not only designed to sense events occurring in the deployment area; they can also be used to detect intruders that attempt to penetrate in this area. So, the goal of barrier coverage is to guarantee that every intruder crossing the barrier of sensor will be detected. Few works are present in the literature for barrier coverage and connectivity. Nevertheless, we can cite the solutions of [15], [16] and [17]. In [15] a distributed algorithm guaranteeing full coverage and multiple connectivity using mobile sensors node is proposed; therefore, the zone of interest is assumed to be known and authors use a grid based deployment algorithm. [16] provides partial coverage after a centralized and probabilistic deployment. The connectivity in this case is intermittent; meaning that, some of the deployed sensors cannot communicate with the base station. [17] made the assumption that $\mathrm{R} \geq \mathrm{r}$ where $\mathrm{R}$ is the communication radius and $\mathrm{r}$ is the sensing radius in order to ensure full coverage and permanent connectivity after a distributed and deterministic deployment.

\subsection{Point coverage and connectivity}

It is often unnecessary to monitor the whole area in many applications; thus monitoring some specific points is sufficient. Each of this point (called point of interest (PoI)) should therefore be covered by at least one sensor node. [18] assume that PoI are static and 
guarantee temporary coverage and intermittent connectivity with random deployment and distributed algorithm. [19] resolved a similar problem differently, and consider that PoI are not static. [20] and [21] consider the problem of full coverage with permanent connectivity. [20] assume that sensors are deployed in a regular zone; therefore they use a grid based deployment algorithm to cover all the PoI. Contrary to [20], [21] ensures full coverage of the PoI using forced based deployment algorithm. These solutions don't propose a node scheduling activity to minimize the energy consumption of the sensors.

Most of the presented works in this section focus on maximizing the rate of coverage of the zone to be covered and don't really study energy consumption of their algorithms; they don't control activities of the nodes. The solution proposed in this paper will also ensure maximum coverage of the AoI and in addition, will guarantee connectivity between sensors. A schedule algorithm will also be proposed in order to minimize energy consumption of both static and mobile nodes, and both normal nodes and $\mathrm{CH}$. Our schedule algorithm also guarantees that any event occurring in the AoI will be detected and the information will be sent to the base station.

\section{Our contribution}

\subsection{Assumptions and notations}

\subsubsection{Assumptions}

In this work, it was assumed that:

- Sensors are randomly deployed in the AoI and, similarly to [12], the number of static sensors nodes that are initially deployed equals the number of sensor nodes that are required to achieve full coverage as if these nodes were deterministically deployed;

- The base station is positioned at the center of the AoI and few mobile nodes are available and can be used to repair the coverage holes after initial deployment of of the static nodes;

- The entire sensors have the same sensing range and the same communication radius.

\subsubsection{Notations}

In the remainder of this work, we will use some notations that we define in this section:

- $T_{i}$ : Awakening time of a normal node;

- $T_{s}$ : Time used by a normal node to send data to its $\mathrm{CH}$ and receive an acknowledgment;

- $T_{c}$ : Time used by a normal node to send collected data in its cluster;

- $T_{n e}$ : Next awakening time of a normal node;

- $\quad N_{c}$ : Number of sensors of a cluster;

- $T_{S S B} \quad$ : Time after which a CH should send data to the base station; 
- $M_{n}$ : The set of mobile nodes;

- $S_{n} \quad$ : The set of static nodes;

- $R_{c}$ : Communication radius of a sensor;

- $\quad R_{\mathrm{S}}$ : Sensing radius of a sensor;

\subsection{Mathematics models}

We represent the WSN by a graph $G=(V ; E)$, where $V$ represents all nodes of the network and $E$ represents the set of edges giving all possible communications.

\subsubsection{Coverage model}

Let $A$ represent the AoI and $q$ a point located in A. The area covered by a sensor $S_{i} \in V$ is defined as the total area located within $R_{s}$ [1]. Analytically, the area covered by a sensor $S_{i} \epsilon$ $\mathrm{V}$ is given by equation 1 :

$$
\mathrm{C}\left(\mathrm{S}_{\mathrm{i}}\right)=\left\{\mathrm{q} \in \mathrm{A} / \mathrm{d}\left(\mathrm{S}_{\mathrm{i}} ; \mathrm{q}\right) \leq \mathrm{R}_{\mathrm{s}}\right\}
$$

So, the area covered by a set of sensors $S=\left\{S_{1}, S_{2} \ldots S_{k}\right\}$ is analytically defined by equation 2:

$$
\mathrm{C}(\mathrm{S})=\mathrm{UC}\left(\mathrm{S}_{\mathrm{k}}\right), \mathrm{k}=\{1, \ldots,|\mathrm{S}|\}
$$

\subsubsection{Connectivity model}

Let us consider $S_{i}$ and $S_{j}$ two sensors nodes deployed in the AoI. $S_{i}$ and $S_{j}$ are directly connected (one-hop connectivity) if and only if $\mathrm{d}\left(\mathrm{S}_{\mathrm{i}} ; \mathrm{S}_{\mathrm{j}}\right) \leq \mathrm{R}_{\mathrm{c}}$. According to [22], a WSN is considered to be connected if there is at least one path between the sink and each node in the considered area.

\subsubsection{Lifetime model}

Let $M=\left\{S_{1}, S_{2} \ldots S_{n}\right\}$ be the set of nodes of a wireless sensor network; $S_{i} \in M$ a given node with lifetime $T_{i}$. In [1], network lifetime is defined by the duration within the network is deployed and the first node loses all its residual energy. So, if $T_{n}$ is the network lifetime, it is computed as follows (equation 3):

$$
\mathrm{T}_{\mathrm{n}}=\min \mathrm{T}_{\mathrm{i}}\left(\mathrm{S}_{\mathrm{i}} \in \mathrm{M}\right)
$$

\subsection{First stage: area coverage procedure}

The first stage of our solution consists in covering as much as possible the AoI in order to collect a maximum number of information. To achieve this, we proceed as follows:

- Remember the BS is located at the center of the topology;

- $\quad$ The sensors are randomly deployed in the AoI;

- After sensor's deployment, we proceed to the clustering of the network using the 
protocol presented in [23]. In the protocol presented in [23], the BS establishes clusters by emitting omnidirectional messages in order to construct crowns and emissions on angle $\alpha$ in order to construct sections. This time, the value of $\alpha$ is determining by using the formula in [24];

- After the clustering stage, sensors proceed to the identification of its cluster's members using the algorithm in [24];

- We now execute the detection of empty clusters using the solution in [25];

- Every $\mathrm{CH}$ then publishes an ordered list representing the cluster's members to the other members of the clusters with the parameters $\mathrm{T}_{\mathrm{i}}, \mathrm{T}_{\mathrm{s}}$ and $\mathrm{T}_{\mathrm{c}}$.

At the end of the previous steps, each static sensor knows in which cluster it belongs to, and the BS knows the list of empty clusters. We now need to feed the empty clusters with mobile nodes. So, after mobile node's deployment, we proceed as follows:

- $\quad$ All the $\mathrm{CH}$ are awake when mobile nodes are deployed. Then, for each node $\mathrm{u} \in \mathrm{M}_{\mathrm{n}}, \mathrm{u}$ broadcasts a hello message in order to determine the coordinate of the cluster in which it has been deployed;

- For each $\mathrm{CH} v \in \mathrm{S}_{\mathrm{n}}$ receiving the hello message from $\mathrm{u}, \mathrm{v}$ just answers by sending the coordinate of its cluster to $\mathrm{u}$. u can therefore determine the cluster in which it has been deployed by considering the message received with the higher power of the signal;

- The CH can now fall asleep and the mobile nodes remain awake. The BS then broadcasts the list of the empty clusters in the priority order. The priority clusters are those who are closest to the Base station. Here the idea is to find the closest mobile sensor $\mathrm{u}$ to an empty cluster and determine the way to move $\mathrm{u}$ to the empty cluster.

- Let $(i ; j)$ be the coordinates of a mobile node $u$ in the virtual architecture; and $\left(i^{\prime} ; j^{\prime}\right)$ the coordinates of an empty cluster. $u$ can determine the distance separating him from the empty cluster using equation4:

$$
\mathrm{d}=\sqrt{\left(i^{x}-i\right)^{2}+\left(j^{s}-j\right)^{2}}
$$

- Each node $u \in M_{n}$ then sends an acknowledgment to the BS containing its identity (i;j), the list of the coordinates of the empty clusters and the distance separating him with these empty clusters;

- At the reception of all these messages, the BS can determine which mobile node $\mathrm{u}$ will feed an empty cluster $\mathrm{E}_{\mathrm{c}}$ with coordinates (i;j), and sends the lists to the mobile nodes;

- Now the mobile nodes can move to the empty clusters using the solution presented in [25].

The algorithm of this stage is given by algorithm 1 . 


\section{Ml Macrothink}

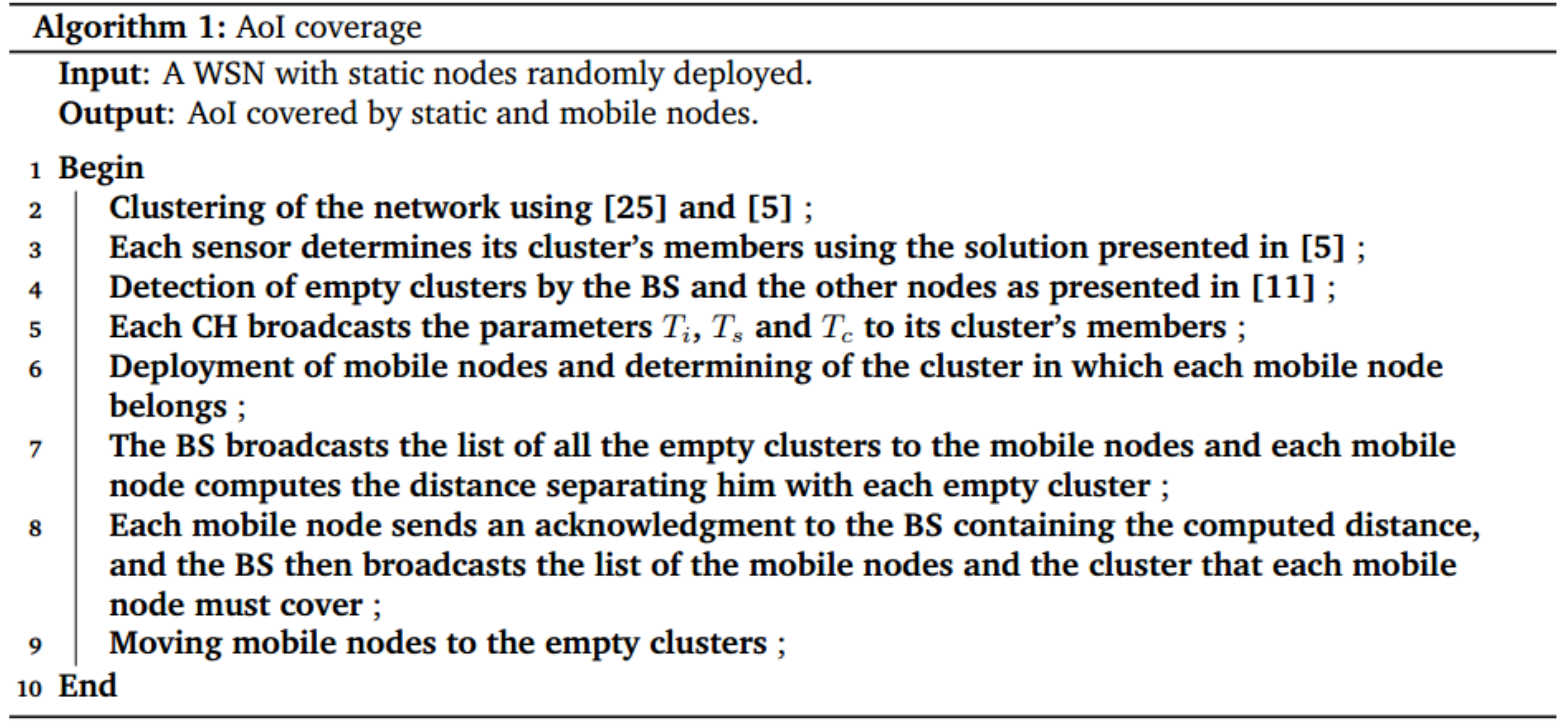

3.4 Second stage: Node scheduling algorithm and sending data to the base station

In this section, we describe how the nodes will be scheduled in order to collect and send data to the base station. Since normal nodes and $\mathrm{CH}$ are scheduled differently, we thus propose two algorithms that will permit us to manage both $\mathrm{CH}$ and normal nodes simultaneously.

\subsubsection{Normal nodes scheduling algorithm and sending data to the $\mathrm{CH}$}

Each node has in its memory the ordered list of his neighbors; so it knows when it should wake up and begin collecting or sending data. According to our notations, a normal node remains awake during $\mathrm{T}_{\mathrm{i}}$. We therefore pose:

$$
\mathrm{T}_{\mathrm{i}}=\mathrm{T}_{\mathrm{s}}+\mathrm{T}_{\mathrm{c}}
$$

So, a normal node executes these instructions when it is awaken:

- It starts by computing the next time after which it should be awaken with the formula: $\mathrm{T}_{\mathrm{ne}}=\left(\mathrm{N}_{\mathrm{c}}-1\right) \mathrm{T}_{\mathrm{i}}$;

- If this node has data collected previously in its memory, it sends it to its $\mathrm{CH}$ and waits for an acknowledgment during the time $\mathrm{T}_{\mathrm{s}}$;

- It remains awake during the time $\mathrm{T}_{\mathrm{c}}$ waiting for an event to occur in the AoI;

- The sensor falls asleep after $\mathrm{T}_{\mathrm{i}}$.

The pseudo-code of our description above is given by the algorithm 2 . 


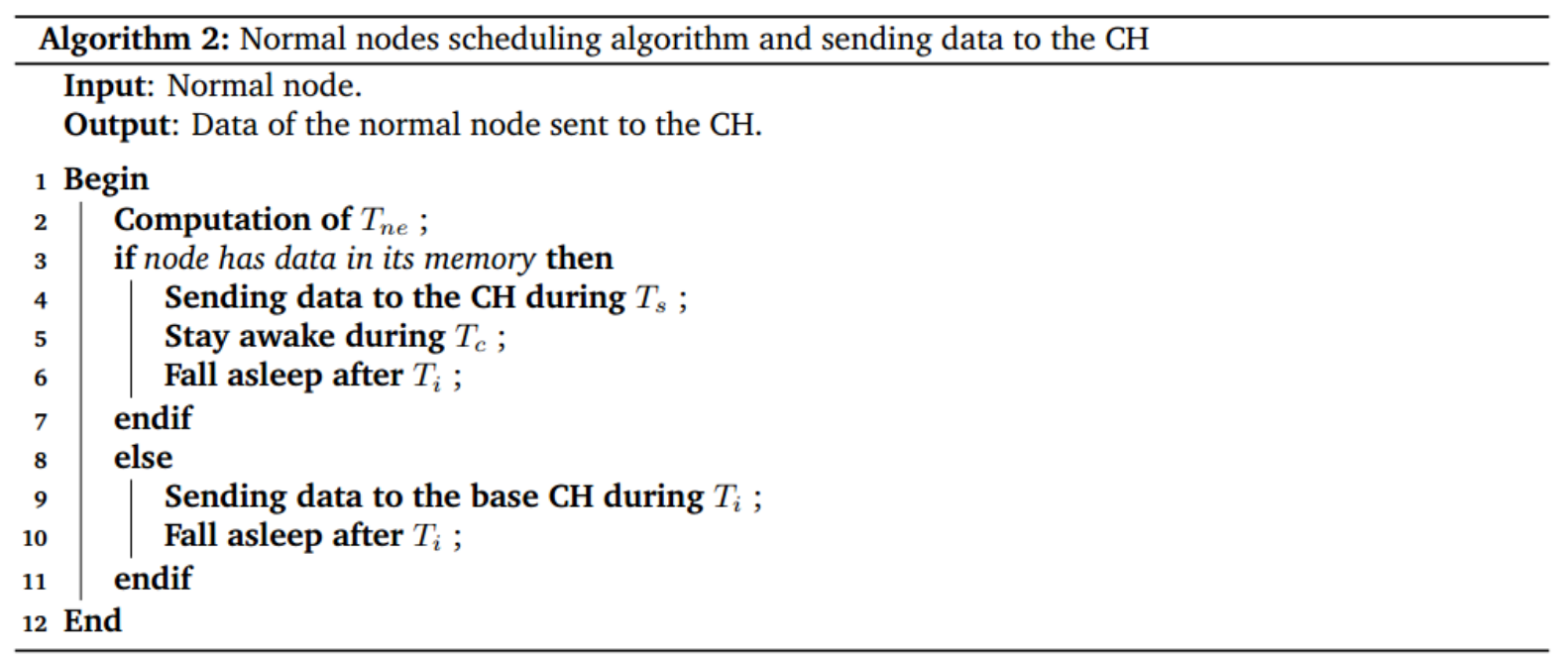

\subsection{2 $\mathrm{CH}$ scheduling algorithm and sending data to the base station}

Our solution recommends that every $\mathrm{T}_{\mathrm{sSB}}$, a $\mathrm{CH}$ must send data to the base station. $\mathrm{T}_{\mathrm{sSB}}$ is computed with the formula:

$$
\mathrm{T}_{\mathrm{sSB}}=\mathrm{N}_{\mathrm{C}} * \mathrm{~T}_{\mathrm{S}}
$$

which means that, after one round of diffusion of its cluster's members, it starts sending data to the base station. Before sending these data, the $\mathrm{CH}$ starts by executing the second part of the DSMAC algorithm [1] which will permit them to synchronize sensors belonging to the path relying the $\mathrm{CH}$ and the base station by sending beacon frames. This will permit us to know all the nodes that will remain awake during the transmission of data to the base station. The $\mathrm{CH}$ can then initiate the transmission. Algorithm 3 describes the pseudo-code of this solution.

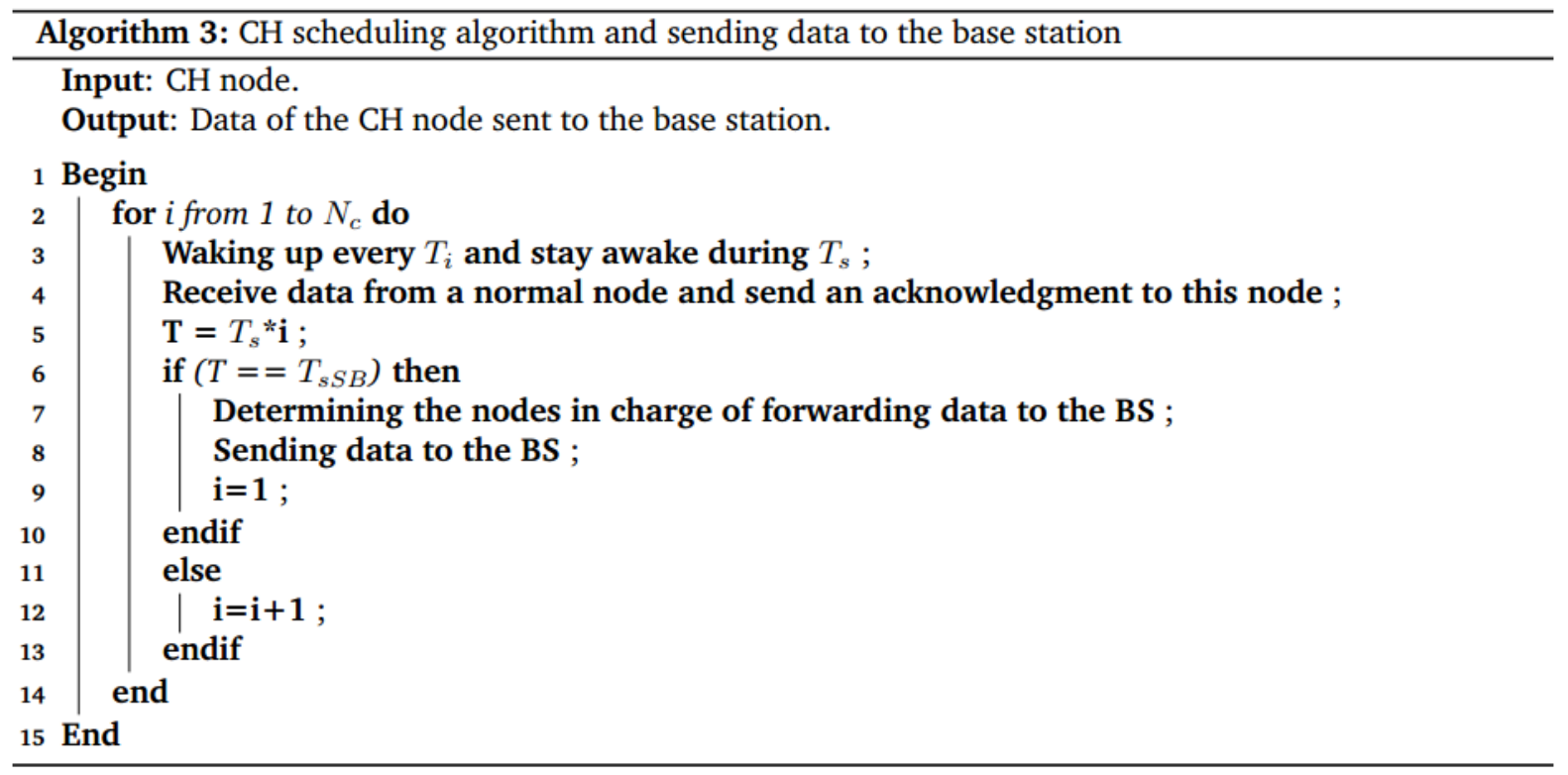

\section{Comparative study of our protocol with some others existing protocols}




\section{Macrothink}

In table 1, we make a comparative study between our protocol and some others.

Table 1. Comparison between our protocol and some others

\begin{tabular}{|l|c|c|c|c|}
\hline \multicolumn{1}{|c|}{ Protocols } & $\begin{array}{c}\text { Deployment } \\
\text { strategy }\end{array}$ & Node scheduling algorithm & $\begin{array}{c}\text { Clustering } \\
\text { algorithm }\end{array}$ & $\begin{array}{c}\text { Use of mobile } \\
\text { nodes }\end{array}$ \\
\hline FCOA [2] & $\begin{array}{c}\text { Random } \\
\text { Semi random and } \\
\text { seterministic } \\
\text { (square based) }\end{array}$ & $\begin{array}{c}\text { Random selection of the } \\
\text { next active node }\end{array}$ & Yes & No \\
\hline DSMAC [4] 26$]$ & $\begin{array}{c}\text { Deterministic } \\
\text { (square based) }\end{array}$ & $\begin{array}{c}\text { Deterministic selection of } \\
\text { the next active node }\end{array}$ & No & No \\
\hline Ammar and al. [27] & $\begin{array}{c}\text { Deterministic } \\
\text { (Grid based) }\end{array}$ & No & No & No \\
\hline Our protocol & Random & $\begin{array}{c}\text { Deterministic selection of } \\
\text { the next active node }\end{array}$ & $\begin{array}{c}\text { Wadaa and al } \\
\text { [23] and } \\
\text { Bomgni and } \\
\text { al. [24] }\end{array}$ & Yes \\
\hline
\end{tabular}

\section{Performance evaluation}

To validate the proposed approach, some simulations were undertaken. The network is heterogeneous, because of the use of both static and mobile nodes. Static sensors have the same deployment parameters such as the sensing and communication radius. We performed extensive simulations by considering the same scenarios and the same parameters. The following curves are the result of at least 100 experiments. In our implementation, the MAC layer is managed in such a way that a node can only receive one message at a time.

\subsection{Coverage ratio of static nodes vs coverage ratio of static and mobile nodes}

The first stage of our contribution consists in finding an optimal solution to cover as much as possible the AoI using static and mobile nodes. Figure 1 presents the coverage ratio of the network when only static nodes are deployed and the coverage ratio of the network when both static and mobile nodes are deployed. 


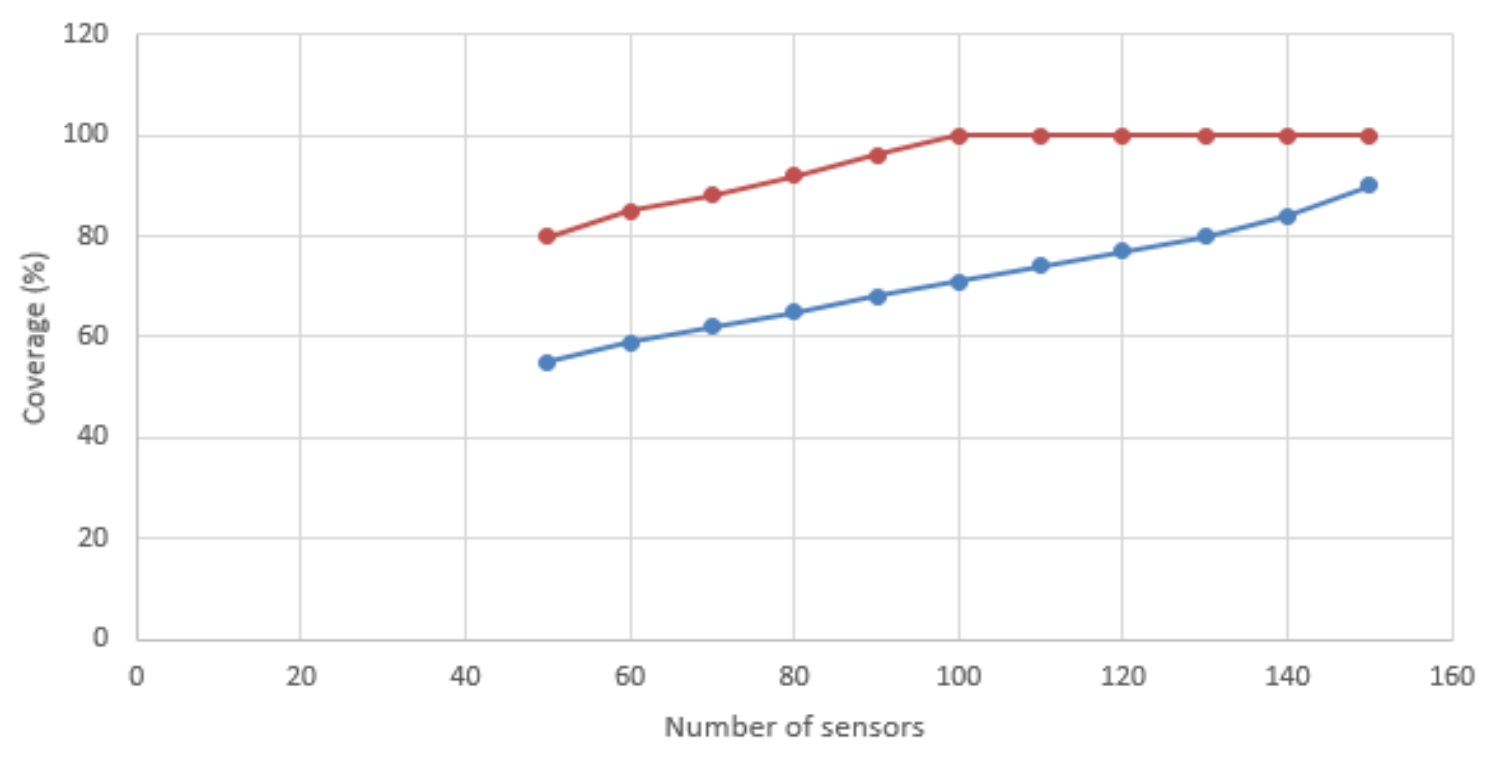

$\rightarrow$ Coverage of static nodes $\rightarrow$ Coverage of static and mobile nodes

Figure 1. Coverage ratio.

\subsection{Energy evaluation of the different type of nodes}

It is very important to prove the efficiency of our protocol by putting forward the energy consumption of the different type of nodes used. In fact we have static nodes divided in two categories (normal nodes and $\mathrm{CH}$ ) and mobile nodes. Due to movements within the network and the fact that they manage one cluster, mobile nodes are nodes which are more expensive in term of energy. Similarly, because of the different tasks achieved by the $\mathrm{CH}$, they consume a lot of energy; but less than the mobile nodes because they don't move. Finally, static sensors are the sensors with the less charge of work. Figure 2 illustrates graphically what we are explaining.

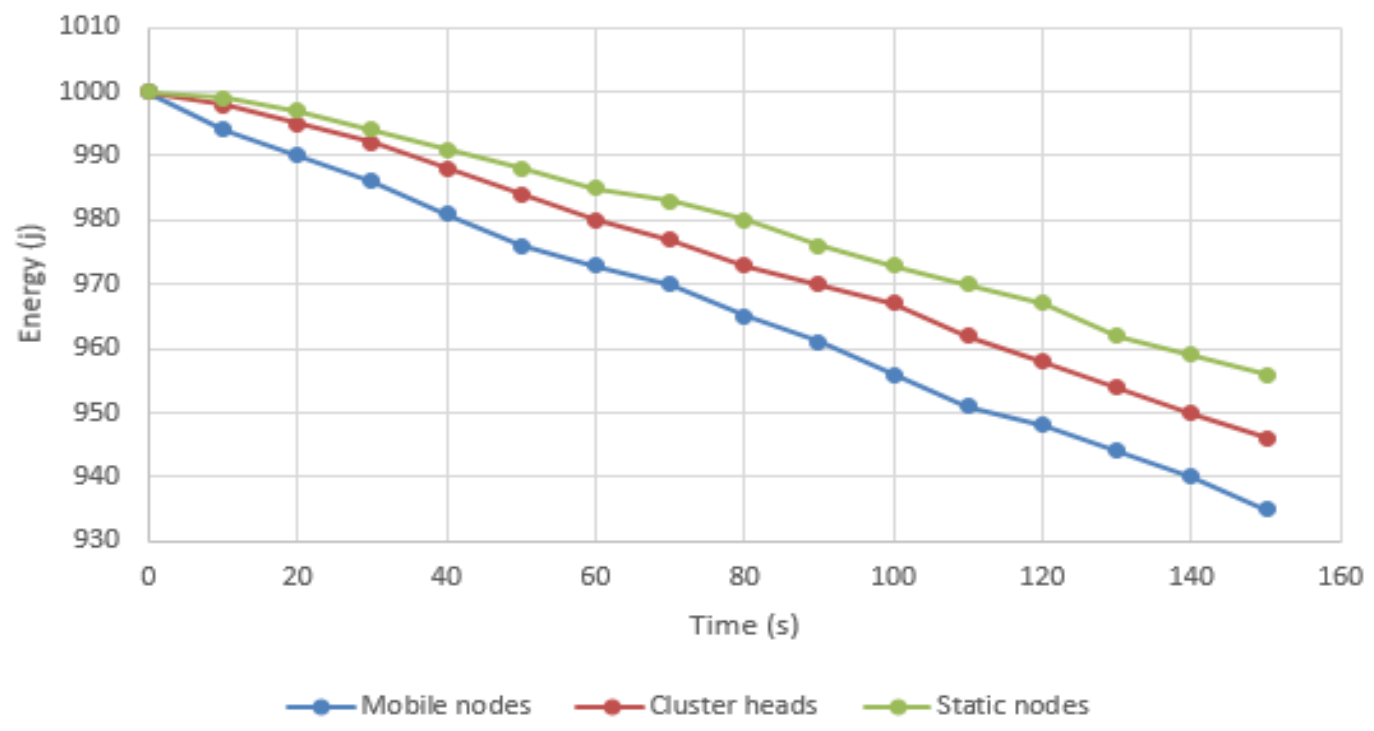

Figure 2. Different node's energy consumption. 


\subsection{Network's lifetime}

We compared the efficiency of our protocol with three other protocols named Flower Pollination Coverage Optimization approach (FPCOA) [2], Semi-Random Deployment Protocol (SRDP) [4] in terms of energy consumption and DSMAC [26]. The results are shown in figure 3.

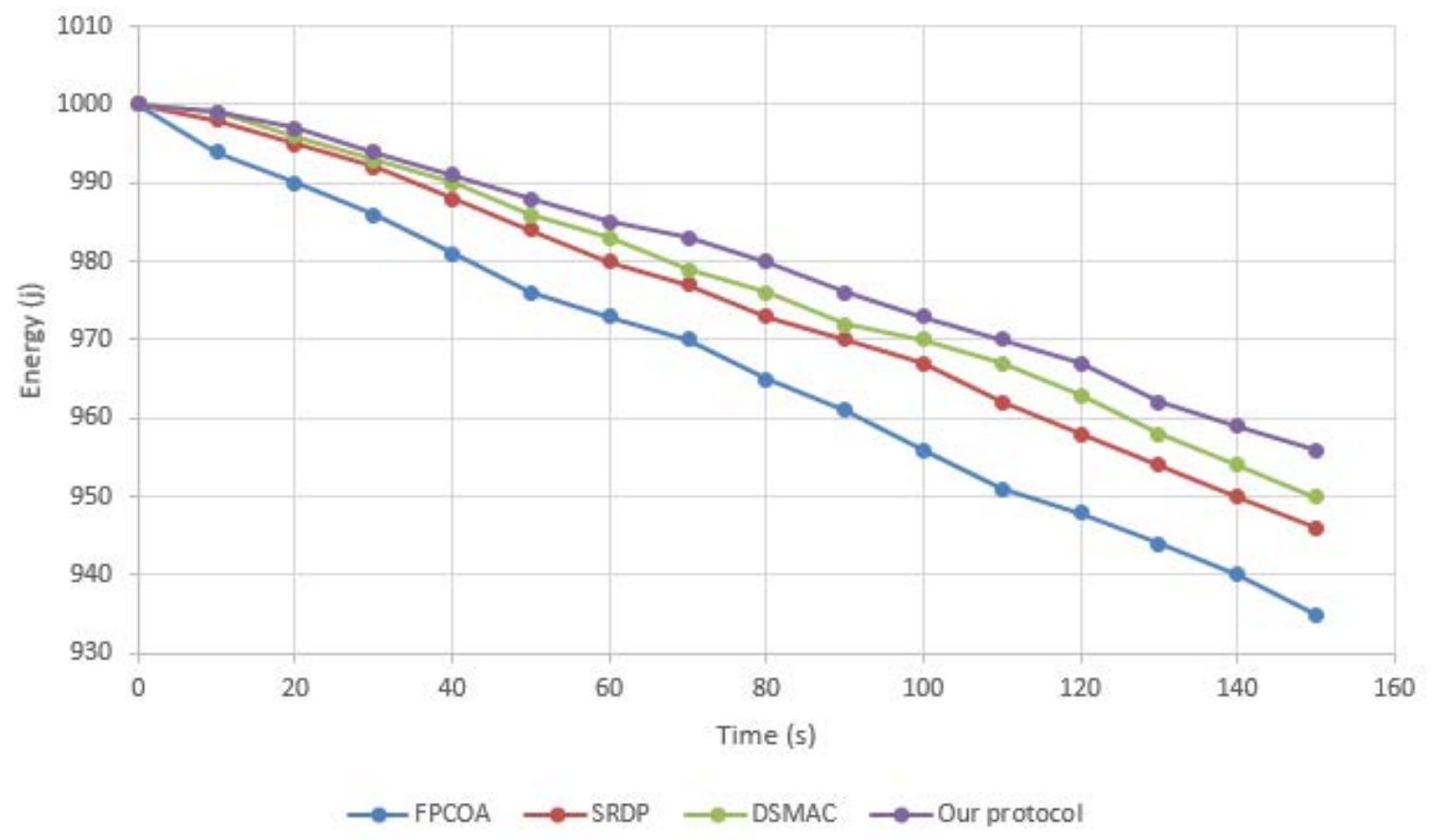

Figure 3. Network's lifetime.

Our protocol is clearly better than the SRDP, FPCOA and DSMAC in term of energy consumption. Since FPCOA doesn't use a clustering scheme to maintain connectivity and reduce energy's consumption of the sensors while exchanging messages, it consumes more energy. The SRDP protocol certainly uses a clustering algorithm, but the latter is not really efficient. In fact, clusters are formed by exchanging hello messages between $\mathrm{CH}$ and its members. Furthermore, this protocol guarantees connectivity and data harvest by randomly activating a sensor which will collect data in the cluster each time. The fact that the active sensor is determined randomly after a computation of a random parameter $P$ consumes more energy at each time that a sensor has to be activated.

\subsection{Average packets received by the sink}

Figure 4 shows the average packets received by the Sink. Figure 4 illustrates that our protocol outperforms DSMAC, SRDP and FPCOA according to the number of packets received by the Sink. The main reason is due to the fact that our protocol avoids collisions because of the implementation of the CSMA/CA protocol and is based on DSMAC algorithm which mitigates the number of collisions. 


\section{Macrothink}

2020

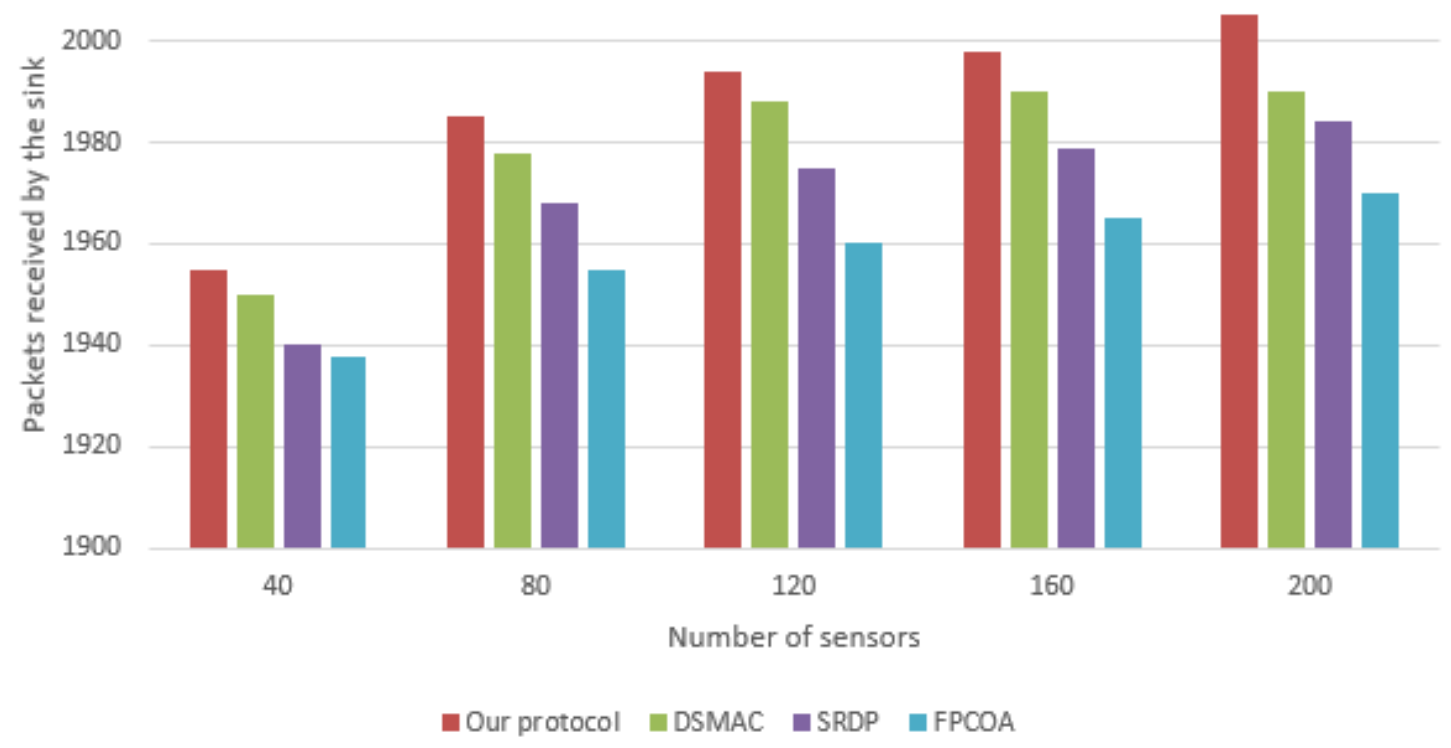

Figure 4. Average packets received by the sink.

\section{Conclusion}

In this paper, we propose a solution named "Area Coverage, Connectivity and Data Collection in wireless sensor networks (A2CC)". The goal of this solution is to cover as much as possible the area of interest (AoI), monitor events occurring in this area and then send data harvested in this area to the base station. To achieve this, we firstly present our approach to guarantee full coverage of the AoI with both static and mobile sensors, and secondly, we propose algorithms to schedule normal nodes and $\mathrm{CHs}$ during the phase of collecting data in the monitored area and the phase of sending data to the base station. Because of the use of CHs and a schedule algorithm the results of our simulations are good and better than many other solutions. In fact, the proposed approach has been compared with several other approaches in the literature in term of energy consumption and average of packets received by the BS. Experiments show that our solution is better than the other approaches, guarantees connectivity and avoid collision of messages.

Despite these encouraging results, several open problems remain. In our future work, we must study how to replace a mobile node whose has completely consumed its residual energy. In fact, if a mobile sensor loses all its residual energy, it remains inactive and therefore, empty clusters (holes) appear in the AoI. We also plan to make the mobile nodes patrol when the number of mobile sensors deployed is less than the empty clusters. In our solution, we have supposed that we always have sufficient number of mobile nodes and we did not manage the case where the number of empty clusters is greater than the number of mobile nodes. 


\section{References}

[1] Ngom, D. (2016). Optimisation de la durée de vie dans les réseaux de capteurs sans fil sous contraintes de couverture et de connectivité réseau. Phd thesis, réseaux et télécommunications, Université de haute Alsace-Mulhouse

[2] Hajjej, F., EjBali, E., \& Zaied, M. (2016). An efficient deployment approach for improved coverage in wireless sensor networks based on flower pollination algorithm. Computer science information technology (CSIT), 117-129, http://dx.doi.org/10.5121/csit.2016.61511

[3] Al-Karaki, J. N., \& Kamal, E. (2004). Routing techniques in wireless sensor networks: a survey. IEEE wireless commun, 11, 6-28, http://dx.doi.org/10.1109/MWC.2004.1368893

[4] Boualem, A., Dahmani, Y., Maatoug, A., \& De-runz, C. (2018). Area coverage optimization in wireless sensor network by semi-random deployment. In proceedings of the $7^{\text {th }}$ international conference on sensor networks (SENSORNETS 2018), 85-90, http://dx.doi.org/10.5220/0006581900850090

[5] Al-Karaki, J. N., \& Gawanmeh, A. (2017). The optimal deployment, coverage, and connectivity problems in wireless sensor networks: revisited. IEEE, 1851-1865, http://dx.doi.org/10.1109/ACCESS.2017.2740382

[6] Khoufi, I., Minet, P., Laouiti, A., \& Mahfoudh, S. (2017). Survey of deployment algorithms in wireless sensor networks: coverage and connectivity issues and challenges. International journal of autonomous and adaptative communications systems (IJAACS), 341-390, http://dx.doi.org/10.1504/IJAACS.2017.088774

[7] Mulligan, R., \& Ammari, H. M. (2010). Coverage in wireless sensor networks: a survey. Networks protocols and algorithms, 2

[8] Ghosh, A., \& Das, S. K. (2008). Coverage and connectivity issues in wireless sensor networks: a survey. Pervasive and mobile computing, 4, 303-334, http://dx.doi.org/10.1016/j.pmcj.2008.02.001

[9] Fan, G., \& Jin, S. (2010). Coverage problem in wireless sensor networks: a survey. Journal of networks, 5, http://dx.doi.org/10.4304/jnw.5.9.1033-1040

[10] Shu, L., Zheng, C., \& Han, G. (2012). A survey on coverage and connectivity issues in wireless sensor networks. Journal of network and computer applications, 619-632

[11] Akewar, M., \& Thkur, N. (2012). Study of wireless mobile network deployment. International journal of computer and wireless communication, 2, 619-632

[12] Banimelhem, O., Mowafi, M., \& Aljoby, W. (2013). Genetic algorithm based node deployment in hybrid wireless sensor networks. Communications and network, 5, http://dx.doi.org/10.4236/cn.2013.54034

[13] Kim, Y. H., Kim, C. M., Yang, D. S., Oh, Y. J., \& Han, Y. H. (2012). Regular sensor deployment patterns for p-coverage and q-connectivity in wireless sensor networks. ICOIN, http://dx.doi.org/10.1109/ICOIN.2012.6164394 


\section{Mll Macrothink}

Network Protocols and Algorithms

ISSN 1943-3581

2018, Vol. 10, No. 4

[14] Yunand, Z., Baiand, X., Xuand, D., Lai, D. H., \& Jia, W. (2010). Optimal deployment patterns for full coverage and k-connectivity $(\mathrm{K}<6)$ wireless sensor networks. IEEE transaction networking, 18

[15] Saipulla, A., Liu, B., Xing, G., \& Wang, J. (2010). Barrier coverge with sensor of limited mobility. MobiHoc, USA

[16] Shibo, H., Jiming, C., Xu, L., Xueming, S. \& Youxian, S. (2012). Cost-effective barrier coverage by mobile sensor network. IEEE INFOCOM

[17] Kong, L., Zhu, Y., Wu, M. Y., \& Shu, W. (2012). Mobile barrier coverage for dynamic objects in wireless sensor networks. Mobile ad hoc and sensor systems MASS, Las vegas, http://dx.doi.org/10.1109/MASS.2012.6502499

[18] Erdelj, M., Natalizio, E., \& Razafindralambo, I. (2012). Multiple point of interest discovery and coverage with mobile wireless sensors. Computing networking and communications ICNC, http://dx.doi.org/10.1109/ICCNC.2012.6167394

[19] Cheng, W., Li, M., Liu, K., Liu, Y., Li, X. \& Liao, X. (2011). Sweep coverage with mobile sensors. Mobile computing IEEE transactions, 10

[20] Li, X., Frey, H., Santoro, N., \& Stojmenovic, I. (2011). Strictly localized sensor self-deployment for optimal focused coverage. IEEE tranasctions on mobile computing, 11, http://dx.doi.org/10.1109/TMC.2010.261

[21] Erdelj, M., Razafindralambo, T., \& Ryl, S. D. (2013). Covering points of interest with mobile sensors. IEEE transaction on parallel and distributed systems, 32-43, http://dx.doi.org/10.1109/TPDS.2012.46

[22] Ngom, D., Lorenz, P., \& Gueye, B. (2015). A distributed scheduling algorithm to improve lifetime in wireless sensor network based on geometric placement of sensors with coverage and connectivity constraints. SENSORCOMM 2015: The Ninth International Conference on Sensor Technologies and Applications, 57-63

[23] Wadaa, A., Olariu, S., Wilson, L., \& Eltoweissy, M. (2005). Training a wireless sensor networks. Mobile networks and applications, 10, 151-168, http://dx.doi.org/10.1023/B:MONE.0000048552.15853.c2

[24] Bomgni, A. B., Jagho, G. B., Fute, E. T., \& Tayou, C. D. (2018). Secure and energy efficient permutation routing protocol for wireless sensors network deployed in space (3D). International journal of computer science and information security, 16, 12-19

[25] Faye, S., \& Myoupo, J. F. (2012). Deployment and management of sparse sensor-actuator network in a virtual architecture. International journal of advanced computer science, 2, 470-477

[26] Ngom, D., Lorenz, P., \& Gueye, B. (2017). DSMAC: constraints-based coverage and connectivity for optimizing the network lifetime in wireless sensor networks.

[27] Ammar, H., XingFu, W., Naji, H. \& Saleem, K. (2014). Grid Coverage Algorithm \& 
Analysis for Wireless Sensor Networks. Network Protocols and Algorithms, 2, 1-19

\section{Copyright Disclaimer}

Copyright for this article is retained by the author(s), with first publication rights granted to the journal.

This is an open-access article distributed under the terms and conditions of the Creative Commons Attribution license (http://creativecommons.org/licenses/by/3.0/). 